\title{
Interconnected vehicle suspension
}

\author{
M C Smith ${ }^{1 *}$ and G W Walker ${ }^{2}$ \\ ${ }^{1}$ Department of Engineering, University of Cambridge, Cambridge, UK \\ ${ }^{2}$ The Math Works, Cambridge, UK
}

The manuscript was received on 22 September 2003 and was accepted after revision for publication on 6 September 2004.

DOI: 10.1243/095440705X6578

\begin{abstract}
This paper introduces a class of passive interconnected suspensions, defined mathematically in terms of their mechanical admittance matrices, with the purpose of providing greater freedom to specify independently bounce, pitch, roll, and warp dynamics than conventional (passive) suspension arrangements. Two alternative realization schemes are described that are capable of implementing this class (under ideal assumptions). The first scheme incorporates an interconnected multilever arrangement consisting of four separate hydraulic circuits, which transforms the separate wheel station displacements to bounce, pitch, roll, and warp motions. Four separate mechanical admittances are connected across the transformed terminals of the multilever. The second scheme is kinematically equivalent to the first but the multilever part consists of four modular subsystems to achieve the same kinematic transformation. The purpose of the class is to allow a high degree of independence between the modes of vehicle motion, e.g. low warp stiffness independent of front and rear anti-roll stiffness. Practical issues that might be involved in implementing the realization schemes are discussed, as well as generalizations to two- and six-wheeled vehicles.
\end{abstract}

Keywords: low warp suspension, hydraulic multi-lever, mechanical networks

\section{INTRODUCTION}

This paper introduces a class of passive interconnected suspensions, defined in terms of their four-port mechanical admittance matrices, with the purpose of providing greater freedom to independently specify bounce, pitch, roll, and warp dynamics than conventional (passive) suspension arrangements. A feature of the class is that several desirable objectives may be achieved together, e.g. low warp stiffness independent of front/rear roll stiffnesses and increased anti-pitch stiffness independent of bounce response. In contrast, a number of the existing approaches to achieve low warp stiffness do so at the expense of roll resistance being provided predominantly at only one end (front or rear) of the car, which has a particular disadvantage for handling.

Two contrasting methods of realization are described which are capable of implementing this class under ideal modelling assumptions. In the first approach, the usual parallel spring-dampers at each wheel station, anti-roll bars, etc., are replaced with a

* Corresponding author: Department of Engineering, University of Cambridge, Cambridge, CB2 1PZ,UK.email: mcs@eng.cam.ac.uk 'mechanical transformer' (multilever), which connects the wheel stations to four mechanical admittances (e.g. parallel spring-dampers). The mechanical transformer consists of four independent hydraulic circuits that serve to transform the combined wheel station motions into generalized deflections, which may represent bounce, pitch, roll, and warp motions, etc.

In the second realization approach, a method is described to replace the hydraulic multilever with a modular system consisting of four subcomponents, which are separately realized. In principle this is dynamically equivalent to the first arrangement, but it allows alternative possibilities in terms of packaging on the vehicle. For example, the subcomponents may be separately located within the vehicle. Alternatively, they may be combined together into one central suspension unit. In addition, the subcomponents may be hydraulically or mechanically realized.

The subject of interconnected vehicle suspensions is by no means a new one, and indeed a variety of different approaches has been reported in the literature. A review and comparison will be given in section 2.2. The ideas of the present paper will be presented here, mainly in the context of a four-wheel 
vehicle, though generalizations to vehicles with any number of wheels or axles is straightforward. This will be illustrated by a generalization to a six-wheel vehicle [e.g. a heavy goods vehicle (HGV) tractor unit], as well as a specialization to a half-car (two-wheel) situation. Practical issues involved in implementing the realization schemes are discussed.

\section{INTERCONNECTED SUSPENSION SCHEMES}

\subsection{Modes of motion}

A (rigid) vehicle body has three principal modes to be controlled (bounce, pitch, and roll). In addition there are the movements of the wheels relative to the body, and here there are four degrees of freedom. It is common to consider the latter in transformed coordinates. When all (relative) displacements are in phase this corresponds to the bounce (wheel) mode. When the front displacements are in phase with each other but out of phase with the rear, this is the pitch mode, and similarly with the roll mode. Finally, there is a case where the front left and rear right displacements are in phase with each other but out of phase with the opposite diagonal-this is usually termed the warp mode [1].

It has long been recognized that there is no real need for the warp mode to be stiffly sprung (one of the earliest references to elucidate this goal is reference [2]). However, standard schemes (e.g. separate spring/damper units at each of the four wheel stations, front/rear anti-roll bars, anti-pitch mechanisms) do not differentiate between roll and warp modes. Further, the use of anti-roll bars, etc., affects only the springing of the suspension and not the damping, so the overall damping characteristic of the suspension system will be a compromise. In the next section some of the schemes that have been proposed to eliminate such compromises will be reviewed.

\subsection{Approaches to suspension interconnection}

Any suspension system where displacements at one wheel station can give rise to forces at other wheel stations can be described as interconnected. In this sense, anti-roll bars are the simplest and most common means of interconnection. Early examples of interconnections between the front and rear of the car were the Citroen 2CV interconnected coil springs and Moulton's hydrolastic suspension [3]. Both schemes act in a similar way to allow the pitch stiffness to be reduced. In the case of the hydroelastic suspension a fluid interconnection along each side of the car tends to produce a compression at one end of the car in response to an extension at the other. This gives a softer ride over bumps, but causes problems with pitch attitude control of the vehicle under braking or variations of the centre of gravity position. These problems have been tackled in several ways, e.g. using rising-rate springs or active self-levelling.

Ortiz [4] has classified a single wheel-pair interconnection as either anti-oppositional or antisynchronous. An anti-roll bar is of the former type, whereas third springs or Z-bars are of the latter type. (The fluid connection of the Moulton scheme can be interpreted as a Z-bar.) It is pointed out in reference [4] that any of the six ways of connecting a single wheel-pair will always stiffen two out of the four modes of motion (bounce, pitch, etc.), leaving the other two unaffected. In general there is a problem in combining such devices because of the need to implement damping separately for each mode as well. This problem is well known even when conventional springs and dampers are used at each wheel station together with anti-roll bars since the dampers need to be chosen as a compromise between optimal settings for roll and bounce. In reference [4] an integrated mechanical/hydraulic scheme is suggested as a possible remedy. The system decouples the four modes into a combined bounce/pitch motion and a combined warp/roll motion, each of which has an associated spring and damper. Additionally, there are units that only resist pitch and roll motions, and serve also to maintain positive system pressure. The system does not appear to completely decouple the four modes of motion, and also the hydromechanical implementation might have packaging problems in some vehicles.

An interesting scheme that achieves low warp stiffness was proposed by Automotive Products [5]. It utilizes four hydraulic actuators, one at each wheel station, with those at the front (rear) being single (double) actuating, or vice versa. In this description it is assumed that there are double-acting actuators at the rear of the vehicle. The tops of the rear actuators are connected via fluid dampers to (a) each other and (b) a gas spring. The undersides of the rear actuators are connected to the opposite front suspension unit, consisting of a hydraulic actuator in series with a parallel gas spring/fluid damper. Thus there are three separate hydraulic circuits and three suspension springs. Although an insightful use of active control gave a good performance for both ride and handling, the soft warp springing was achieved by virtue of the hydraulic hardware interconnections. This has an appealing simplicity, but 
there is the disadvantage that the springing and damping of the four modes cannot be specified independently of one another in this manner and the front-rear anti-roll balance is restricted. A purely passive scheme of a similar type has also been proposed in reference [6].

The use of hydraulic interconnections to provide a suspension system with low warp resistance has been exploited in several related schemes from Kinetic Limited (see, for example, reference [7]). These typically employ multiple single- or double-acting pistons at each wheel station together with a number of longitudinal and cross-car couplings to achieve some separate control of the vehicle modes of motion. Multiple accumulator units are incorporated within the hydraulic circuits to provide a springing capability.

\subsection{Active schemes}

The problems of (a) ride - to insulate a vehicle body from road undulations and (b) handling - to restrain vehicle body motions in response to inertial loads (such as those resulting from braking and cornering) are well known to be conflicting requirements for passive suspensions. The fact that active suspensions can remove the compromise between these goals has also been well recognized (see, for example, references $[\mathbf{5}]$ and $[\mathbf{8}]$ to $[\mathbf{1 0}]$ ). The additional complexity required to implement 'fully active' suspension systems can also be put to good use to achieve other desirable characteristics of advanced passive suspensions, such as those described in section 2.2. Active schemes that specifically decompose the strut displacement signals into their modal components (e.g. bounce, roll, etc.) have been explicitly described (see, for example, references $[\mathbf{8}]$ and [11]). An alternative strategy has been active augmentation of schemes such as those described in section 2.2 (e.g. references [5] and [7]).

\subsection{Definition of a class of interconnected suspensions}

An approach to understand fundamental limitations in suspension systems using mechanical multiport networks was put forward in reference [9]. This viewpoint was applied both to the full vehicle and to the suspension system. In the present context the viewpoint gives a useful abstraction of an interconnected suspension system (for a four-wheeled vehicle) as a multiport network, as shown in Fig. 1, where the velocity/force pairs, $\left(v_{\mathrm{lf}}, F_{\mathrm{lf}}\right), \quad\left(\nu_{\mathrm{rf}}, F_{\mathrm{rf}}\right)$, $\left(v_{\mathrm{lr}}, F_{\mathrm{lr}}\right)$ and $\left(v_{\mathrm{rr}}, F_{\mathrm{rr}}\right)$ represent the four relative velocities between the wheel hub and vehicle body

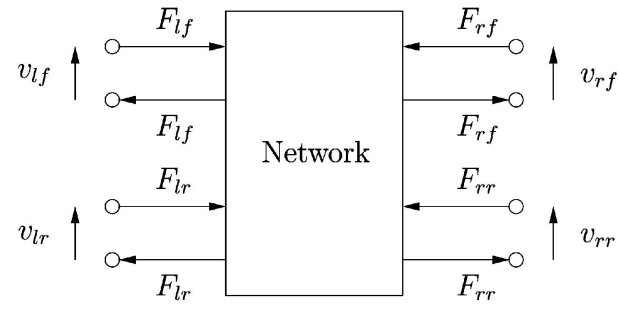

Fig. 1 A mechanical multiport network

and equal-and-opposite suspension force pairs at the left-front, right-front, left-rear, and right-rear of the vehicle. Figure 1 is motivated by the force-current mechanical-electrical analogy, which interprets force and current (respectively velocity and voltage) as through (respectively across) variables [12].

For the network representation of Fig. 1 the admittance of the suspension system can be defined as the matrix $\mathbf{Y}(s)$, which connects the Laplace-transformed velocities and forces as follows

$$
\left[\begin{array}{c}
\hat{F}_{\text {lf }} \\
\hat{F}_{\text {rf }} \\
\hat{F}_{\text {lr }} \\
\hat{F}_{\text {rr }}
\end{array}\right]=\mathbf{Y}(s)\left[\begin{array}{c}
\hat{v}_{\text {lf }} \\
\hat{v}_{\text {rf }} \\
\hat{v}_{\mathrm{lr}} \\
\hat{v}_{\text {rr }}
\end{array}\right]
$$

As an illustration it is noted that the admittance matrix of a conventional suspension arrangement has the form

$$
\mathbf{Y}_{\mathrm{c}}=\left[\begin{array}{cccc}
q_{1}+q_{2} & -q_{2} & 0 & 0 \\
-q_{2} & q_{1}+q_{2} & 0 & 0 \\
0 & 0 & q_{3}+q_{4} & -q_{4} \\
0 & 0 & -q_{4} & q_{3}+q_{4}
\end{array}\right]
$$

where $q_{1}=k_{\mathrm{f}} / s+c_{\mathrm{f}}, \quad q_{2}=k_{\mathrm{af}} / s, \quad q_{3}=k_{\mathrm{r}} / s+c_{\mathrm{r}}$ and $q_{4}=k_{\mathrm{ar}} / s$. The constants $k_{\mathrm{f}}, c_{\mathrm{f}}$ and $k_{\mathrm{af}}\left(k_{\mathrm{r}}, c_{\mathrm{r}}\right.$ and $\left.k_{\mathrm{ar}}\right)$ represent the front (respectively rear) spring, damper, and anti-roll bar rates.

The main purpose of this paper is to introduce a class of interconnected suspensions defined through a class of admittance matrices as follows

$$
\mathbf{Y}(s)=\left(\mathbf{T}^{\prime}\right)^{-1} \operatorname{diag}\left(Q_{1}, Q_{2}, Q_{3}, Q_{4}\right) \mathbf{T}^{-1}
$$

where $\mathbf{T}$ is a real constant non-singular $4 \times 4$ matrix and $Q_{1}(s), \ldots, Q_{4}(s)$ are admittances of passive mechanical one-ports, e.g. a spring and damper in parallel with an admittance $k / s+c$. Alternatively, the class can be described in terms of the impedance matrix $\mathbf{Z}(s)=\mathbf{Y}(s)^{-1}=\mathbf{T} \operatorname{diag}\left(Q_{1}^{-1}, \ldots, Q_{4}^{-1}\right) \mathbf{T}^{\prime}$. In sections 3 and 5 two contrasting approaches to realize this class will be presented. In section 3 the motivation for this class of admittances will be explained in terms of a 
potential to decouple the four principal modes of motion, as well as adjusting the front-rear balance for these modes.

The purpose of introducing this class is twofold. Firstly, by defining a broad class of (ideal) interconnected suspensions mathematically, attention is focused on the degrees of freedom that particular schemes offer. Many existing schemes appear to be a subclass of the one defined and may be restricted in certain ways (e.g. low warp stiffness may be achieved at the expense of anti-roll balance being fixed unfavourably). Secondly, the definition of a class of admittances allows a systematic evaluation of the performance advantage of a particular scheme by means of optimization of desired performance measures for a full-car vehicle model. For reasons of space a full investigation of these directions is deferred to a subsequent work [13].

\section{A HYDRAULIC REALIZATION OF THE INTERCONNECTED SUSPENSION}

\subsection{General description}

The first realization approach for the class of interconnected suspensions described in section 2.4 is illustrated in Fig. 2. The four piston units on the lefthand side of the diagram will be referred to as the actuators. Each is connected between the respective wheel hubs and the vehicle body in place of the usual suspension arrangement, e.g. a parallel springdamper strut. Each actuator is associated with a separate hydraulic circuit. The circuits are each in turn connected to four further cylinders, which are arranged together in groups of four. The corresponding pistons in each group are constrained to move together, and each of these is termed a kinematic constraint mechanism (KCM). To each KCM there corresponds a pair of points or terminals between which a suspension unit is connected.

At its simplest level the arrangement envisages the suspension units to be a spring and damper in parallel. More general scalar admittances $Q_{1}, \ldots, Q_{4}$ (e.g. involving multiple springs and dampers, etc.) could be considered, as well as possible interconnections between the actuators or active elements. The part of the mechanism consisting of the four hydraulic circuits can be thought of as a mechanical transformer between the four actuators and the four suspension units.

To understand the idea behind the arrangement it is useful to consider the case where all the piston areas $A_{\mathrm{lf}}, A_{32}$, etc., are equal. Then, if equal displace- ments are imposed at each actuator (wheel strut), i.e. $\left(x_{\mathrm{lf}}, x_{\mathrm{rf}}, x_{\mathrm{Ir}}, x_{\mathrm{rr}}\right)=(1,1,1,1)$, a displacement occurs at the suspension unit $Q_{1}$ but at no other suspension unit. Similarly, if the wheel strut displacements are $(-1,-1,1,1),(-1,1,-1,1)$, or $(-1,1,1,-1)$, then a displacement occurs only at $Q_{2}, Q_{3}$, or $Q_{4}$ (respectively). Thus, the suspension units 1, 2, 3, and 4 can be associated with bounce, pitch, roll, and warp motions respectively. In contrast, displacements at a single actuator (single-wheel inputs) result in a linear combination of displacements at each suspension unit.

\subsection{Derivation of the suspension admittance matrix}

In Fig. 2 the velocity/force pairs $\left(v_{\mathrm{lf}}, F_{\mathrm{lf}}\right),\left(\nu_{\mathrm{rf}}, F_{\mathrm{rf}}\right)$, $\left(v_{\mathrm{lr}}, F_{\mathrm{lr}}\right)$ and $\left(v_{\mathrm{rr}}, F_{\mathrm{rr}}\right)$ represent the four relative velocities and equal-and-opposite force pairs across the actuators at the left-front, right-front, left-rear and right-rear of the vehicle. The admittance matrix, which is the transfer function from the (Laplacetransformed vectors of) velocity to the force, will be calculated.

It is assumed that the four hydraulic circuits act as ideal levers, i.e. without elasticity, damping, inertia effects, etc. The net flow into each fluid circuit is zero, which gives

$$
\begin{aligned}
& v_{\mathrm{lf}} A_{\mathrm{lf}}+v_{1} A_{11}-v_{2} A_{22}-v_{3} A_{32}-v_{4} A_{42}=0 \\
& v_{\mathrm{rr}} A_{\mathrm{rf}}+v_{1} A_{12}-v_{2} A_{21}+v_{3} A_{32}+v_{4} A_{42}=0 \\
& v_{\mathrm{lr}} A_{\mathrm{lr}}+v_{1} A_{13}+v_{2} A_{22}-v_{3} A_{31}+v_{4} A_{41}=0 \\
& v_{\mathrm{rr}} A_{\mathrm{rr}}+v_{1} A_{14}+v_{2} A_{21}+v_{3} A_{31}-v_{4} A_{41}=0
\end{aligned}
$$

where $v_{i}$ is the relative velocity across the suspension unit $i$ and the remaining constants are the areas of the various pistons. At any point in a hydraulic circuit the pressure must be the same, which gives

$$
\begin{aligned}
& \frac{F_{\mathrm{lf}}}{A_{\mathrm{lf}}}=\frac{F_{11}}{A_{11}}=-p_{1} \\
& \frac{F_{\mathrm{rf}}}{A_{\mathrm{rf}}}=\frac{F_{12}}{A_{12}}=-p_{2} \\
& \frac{F_{\mathrm{lr}}}{A_{\mathrm{lr}}}=\frac{F_{13}}{A_{13}}=-p_{3} \\
& \frac{F_{\mathrm{rr}}}{A_{\mathrm{rr}}}=\frac{F_{14}}{A_{14}}=-p_{4}
\end{aligned}
$$




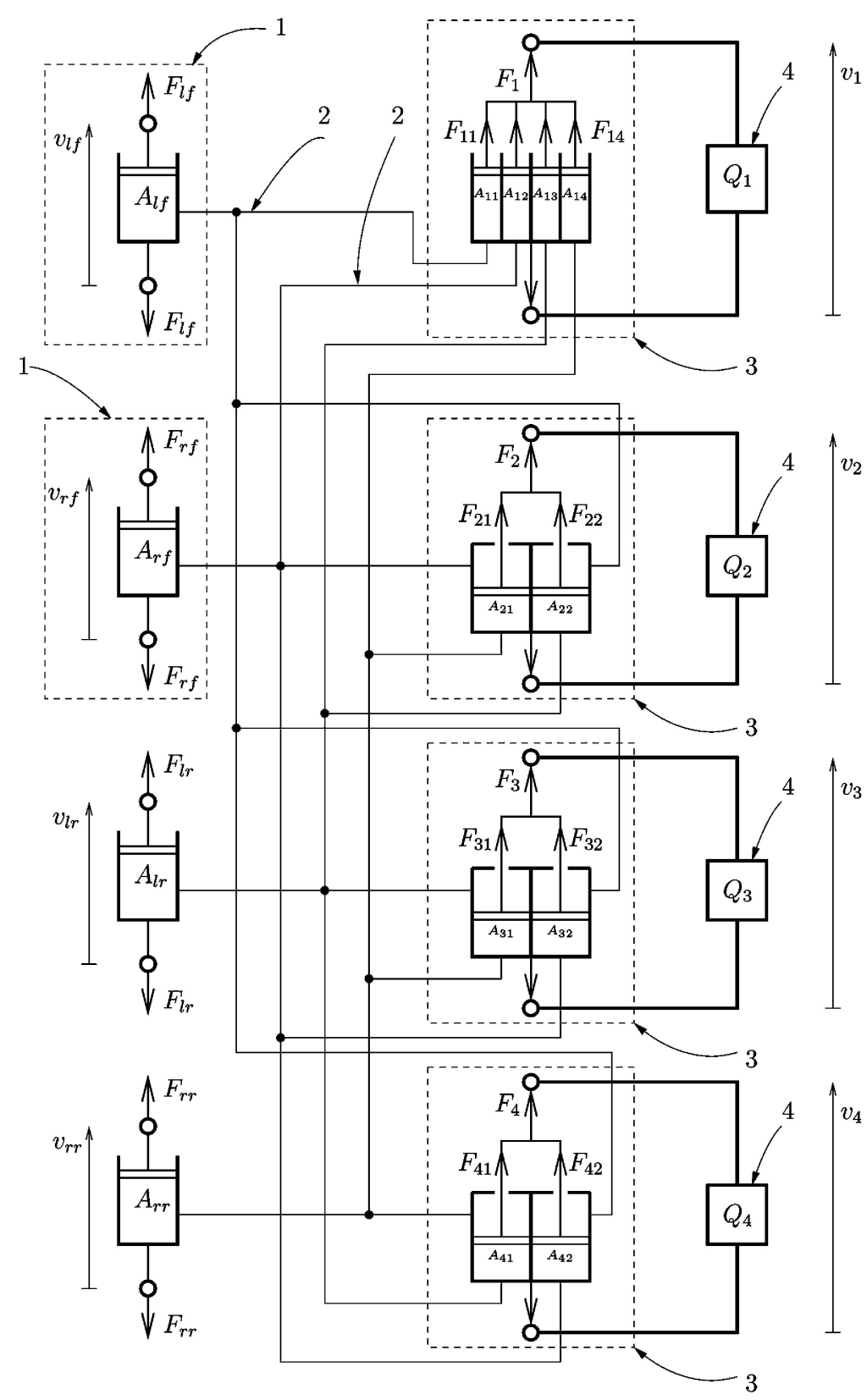

Fig. 2 Interconnected vehicle suspension: four-wheel version (1, actuator; 2, hydraulic circuit; 3 , kinematic constraint mechanism; 4, suspension unit)

where $p_{\mathrm{i}}$ is the pressure in circuit $i$ and $F_{11}$, etc., are the forces indicated. Also

$$
\begin{aligned}
& F_{1}=F_{11}+F_{12}+F_{13}+F_{14} \\
& F_{2}=F_{21}+F_{22} \\
& F_{3}=F_{31}+F_{32} \\
& F_{4}=F_{41}+F_{42}
\end{aligned}
$$

and

$$
\begin{aligned}
& F_{21}=A_{21}\left(p_{2}-p_{4}\right) \\
& F_{22}=A_{22}\left(p_{1}-p_{3}\right) \\
& F_{31}=A_{31}\left(p_{3}-p_{4}\right) \\
& F_{32}=A_{32}\left(p_{1}-p_{2}\right) \\
& F_{41}=A_{41}\left(p_{4}-p_{3}\right) \\
& F_{42}=A_{42}\left(p_{1}-p_{2}\right)
\end{aligned}
$$


Therefore

$$
\left[\begin{array}{c}
v_{\mathrm{lf}} \\
v_{\mathrm{rf}} \\
v_{\mathrm{lr}} \\
v_{\mathrm{rr}}
\end{array}\right]=\mathbf{T}\left[\begin{array}{c}
v_{1} \\
v_{2} \\
v_{3} \\
v_{4}
\end{array}\right], \quad\left[\begin{array}{c}
F_{1} \\
F_{2} \\
F_{3} \\
F_{4}
\end{array}\right]=-\mathbf{T}^{\prime}\left[\begin{array}{c}
F_{1 \mathrm{lf}} \\
F_{\mathrm{rf}} \\
F_{\mathrm{lr}} \\
F_{\mathrm{rr}}
\end{array}\right]
$$

where

$$
\mathbf{T}=\left[\begin{array}{cccc}
-\frac{A_{11}}{A_{\mathrm{lf}}} & \frac{A_{22}}{A_{\mathrm{lf}}} & \frac{A_{32}}{A_{\mathrm{lf}}} & \frac{A_{42}}{A_{\mathrm{lf}}} \\
-\frac{A_{12}}{A_{\mathrm{rf}}} & \frac{A_{21}}{A_{\mathrm{rf}}} & -\frac{A_{32}}{A_{\mathrm{rf}}} & -\frac{A_{42}}{A_{\mathrm{rf}}} \\
-\frac{A_{13}}{A_{\mathrm{lr}}} & -\frac{A_{22}}{A_{\mathrm{lr}}} & \frac{A_{31}}{A_{\mathrm{lr}}} & -\frac{A_{41}}{A_{\mathrm{lr}}} \\
-\frac{A_{14}}{A_{\mathrm{rr}}} & -\frac{A_{21}}{A_{\mathrm{rr}}} & -\frac{A_{31}}{A_{\mathrm{rr}}} & \frac{A_{41}}{A_{\mathrm{rr}}}
\end{array}\right]
$$

The admittances $Q_{1}, \ldots, Q_{4}$ connected across the KCMs imply the equations $\hat{F}_{1}=-Q_{1} \hat{v}_{1}$, etc. (where ^ denotes the Laplace transform), which gives

$$
\left[\begin{array}{c}
\hat{F}_{\text {lf }} \\
\hat{F}_{\text {rf }} \\
\hat{F}_{\text {lr }} \\
\hat{F}_{\text {rr }}
\end{array}\right]=-\left(\mathbf{T}^{\prime}\right)^{-1}\left[\begin{array}{c}
\hat{F}_{1} \\
\hat{F}_{2} \\
\hat{F}_{3} \\
\hat{F}_{4}
\end{array}\right]
$$

$$
\begin{array}{cl}
=\left(\mathbf{T}^{\prime}\right)^{-1} \operatorname{diag}\left(Q_{1}, Q_{2}, Q_{3}, Q_{4}\right)\left[\begin{array}{c}
\hat{v}_{1} \\
\hat{v}_{2} \\
\hat{v}_{3} \\
\hat{v}_{4}
\end{array}\right] & A_{1 \mathrm{f}}=A_{\mathrm{rf}} \\
A_{1 \mathrm{r}}=A_{\mathrm{rr}} \\
{\left[\begin{array}{c}
\hat{v}_{\mathrm{lf}} \\
\hat{v}_{\mathrm{r}}
\end{array}\right.} & A_{11}=A_{12} \\
A_{13}=A_{14} \\
A_{21}=A_{22}
\end{array}
$$

This means that the matrix $\mathbf{T}$ takes the general form

$$
\mathbf{T}=\left[\begin{array}{cccc}
-\lambda_{1} & \lambda_{3} & \lambda_{5} & \lambda_{7} \\
-\lambda_{1} & \lambda_{3} & -\lambda_{5} & -\lambda_{7} \\
-\lambda_{2} & -\lambda_{4} & \lambda_{6} & -\lambda_{8} \\
-\lambda_{2} & -\lambda_{4} & -\lambda_{6} & \lambda_{8}
\end{array}\right]
$$

The ratio $\lambda_{1} / \lambda_{2}$ determines the front-rear weighting associated with the bounce suspension unit. The other ratios, $\lambda_{3} / \lambda_{4}$, etc., determine the front-rear weighting for the pitch, roll, and warp suspension 
units. These ratios are given by the expressions

$$
\begin{aligned}
& \frac{\lambda_{1}}{\lambda_{2}}=\frac{A_{11}}{A_{13}} \frac{A_{1 \mathrm{r}}}{A_{\mathrm{lf}}} \\
& \frac{\lambda_{3}}{\lambda_{4}}=\frac{A_{1 \mathrm{r}}}{A_{1 \mathrm{f}}} \\
& \frac{\lambda_{5}}{\lambda_{6}}=\frac{A_{32}}{A_{31}} \frac{A_{1 \mathrm{r}}}{A_{1 \mathrm{f}}} \\
& \frac{\lambda_{7}}{\lambda_{8}}=\frac{A_{42}}{A_{41}} \frac{A_{\mathrm{lr}}}{A_{1 \mathrm{f}}}
\end{aligned}
$$

It is evident that the full freedom can be obtained by first specifying a desired ratio between the front and rear actuator areas and then choosing the remaining piston areas in the KCMs.

\subsection{Front-rear weighting}

An advantage claimed for the present class of interconnected suspensions is the possibility to adjust the front-rear balance in roll stiffness at the same time as having low warp stiffness. This fact is illustrated by considering the forces provided in response to a steady state roll input defined by

$$
\left[\begin{array}{c}
x_{\mathrm{lf}} \\
x_{\mathrm{rf}} \\
x_{\mathrm{lr}} \\
x_{\mathrm{rr}}
\end{array}\right]=\left[\begin{array}{c}
a \\
-a \\
b \\
-b
\end{array}\right]
$$

for some positive constants $a$ and $b$. Consider an interconnected suspension with admittance given by equation (2), where $\mathbf{T}$ is defined as in equation (10). Suppose that the static stiffnesses of the four suspension units is given by $\left.s\left(Q_{1}(s)\right)\right|_{s=0}=k_{\mathrm{b}},\left.s\left(Q_{2}(s)\right)\right|_{s=0}=k_{\mathrm{p}}$, $\left.s\left(Q_{3}(s)\right)\right|_{s=0}=k_{\mathrm{r}}$ and $\left.s\left(Q_{4}(s)\right)\right|_{s=0}=0$, so that the warp suspension unit has zero static stiffness. Then it is easy to check that

$$
\begin{aligned}
{\left[\begin{array}{c}
F_{\text {lf }} \\
F_{\text {rf }} \\
F_{\text {lr }} \\
F_{\text {rr }}
\end{array}\right] } & \left(\mathbf{T}^{\prime}\right)^{-1} \operatorname{diag}\left(k_{\mathrm{b}}, k_{\mathrm{p}}, k_{\mathrm{r}}, 0\right) \mathbf{T}^{-1}\left[\begin{array}{c}
a \\
-a \\
b \\
-b
\end{array}\right] \\
& =\frac{k_{\mathrm{r}}\left(b \lambda_{7}+a \lambda_{8}\right)}{2\left(\lambda_{5} \lambda_{8}+\lambda_{6} \lambda_{7}\right)^{2}}\left[\begin{array}{c}
\lambda_{8} \\
-\lambda_{8} \\
\lambda_{7} \\
-\lambda_{7}
\end{array}\right]
\end{aligned}
$$

Thus the front-rear balance in roll stiffness is determined by the ratio $\lambda_{8} / \lambda_{7}$ associated with the warp suspension unit.

\subsection{Implementation of the kinematic constraint mechanism}

In this section a version of the hydraulic interconnected suspension will be illustrated, which suggests a possible method that can be used to realize the kinematic constraint mechanisms shown in Fig. 3. Each KCM is implemented by means of a pivoted beam to which are attached four singleacting pistons connected to each of the hydraulic circuits. (For the bounce KCM a four-bar link with two pivots is shown for convenience.) A suspension unit is connected to each beam.

The four KCMs and associated suspension units, and also the interconnecting parts of the hydraulic circuits, are all grouped together in what is called the central suspension unit. This may be a convenient way to implement the suspension concept, with the central suspension unit placed at a convenient location on the vehicle and connected to each wheel station actuator by single hydraulic pipes.

If the perpendicular distances from the central pivots to the suspension units are denoted $d_{1}, d_{2}, d_{3}$ and $d_{4}$ and $\lambda_{1}=\mu_{1} / d_{1}, \lambda_{2}=\mu_{2} / d_{1}, \lambda_{3}=\mu_{3} / d_{2}, \lambda_{4}=\mu_{4} / d_{2}$, $\lambda_{5}=\mu_{5} / d_{3}, \lambda_{6}=\mu_{6} / d_{3}, \lambda_{7}=\mu_{7} / d_{4}$, and $\lambda_{8}=\mu_{8} / d_{4}$, then a check can be made that the suspension admittance is given by equation (2) with $\mathbf{T}$ as in equation (10). In this implementation the front-rear weighting for each suspension unit can be independently adjusted by means of the lever-arm lengths with all the piston areas kept constant.

In Fig. 3 each actuator piston is connected by a bell crank to a rod, which is connected to the wheel hub (not shown). In this example, a pre-load spring is attached to the bell crank to increase hydraulic pressure. Alternatively, pre-load springs may be connected in parallel with the rod between the vehicle body and wheel hub. According to whether the springs are in compression or extension, they serve to increase hydraulic pressure or carry some weight of the vehicle. If the velocity-force pairs $\left(v_{\mathrm{lf}}, F_{\mathrm{lf}}\right)$, etc., are redefined with respect to the rods rather than the actuators, then the mechanical admittance of the suspension system is modified by the addition of a diagonal matrix of admittances associated with the pre-load springs.

For a practical implementation of the suspension system of Figs 2 and 3 ordinary piston-cylinder arrangements have disadvantages, primarily leakage 


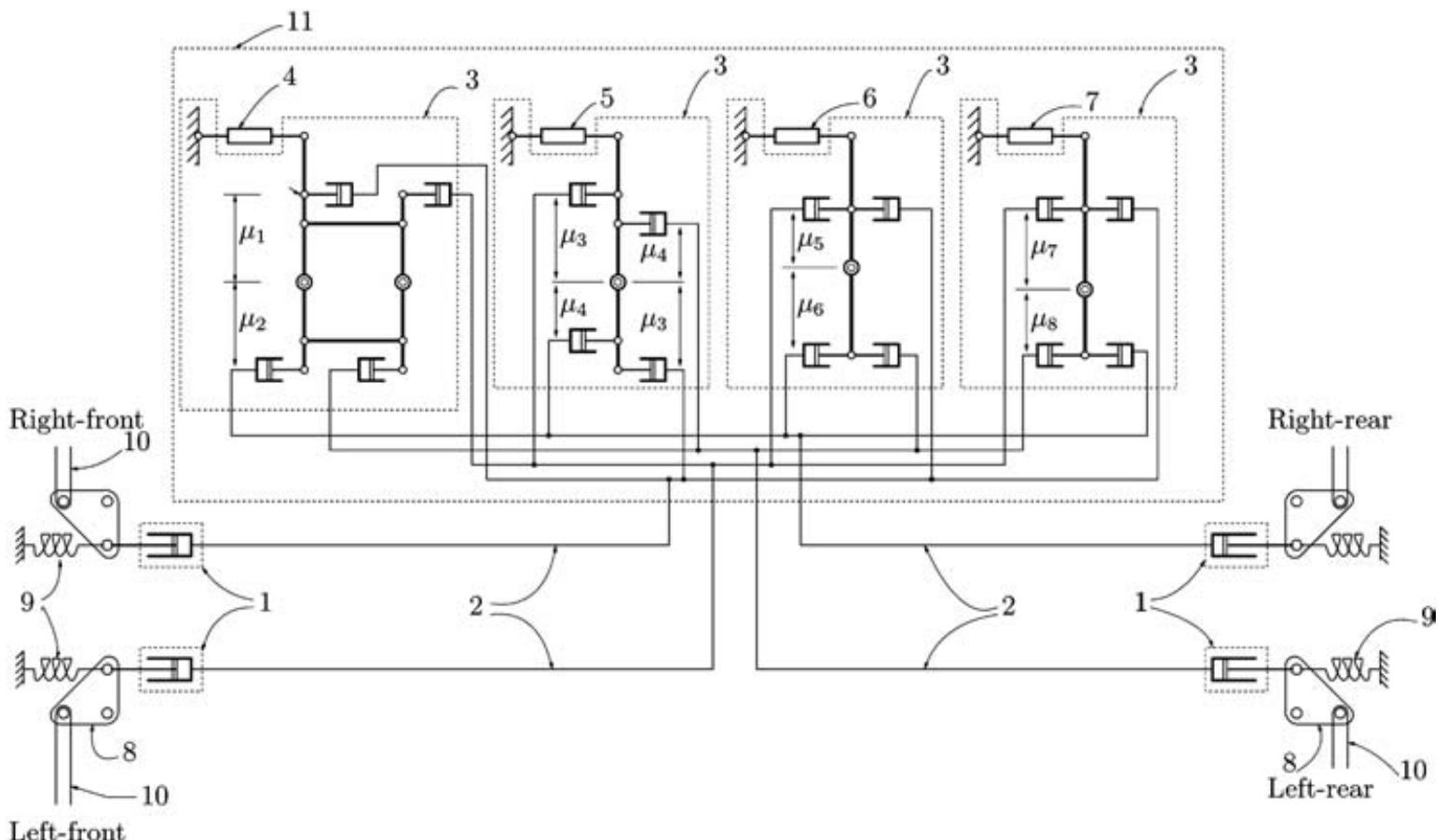

Fig. 3 Interconnected vehicle suspension with realization of kinematic constrain mechanisms (1, actuator; 2, hydraulic circuit; 3, kinematic constraint mechanism; 4-7, suspension units for bounce, pitch, roll, and warp; 8, bell crank; 9, pre-load spring; 10, suspension rod; 11, central suspension unit)

and friction. Drawing on currently available suspension designs, an alternative to the piston-cylinder is presented in Fig. 4. This is based on the standard oleo-pneumatic device employed in the Moulton hydroelastic system and in various Citroen schemes, and employs a flexible membrane between the working fluid and the piston, thus avoiding the leakage problems associated with a conventional piston.

It is worth noting that the load at each strut caused by the weight of the vehicle will ensure a positive pressure in each hydraulic circuit in the steady state. This has the advantage of reducing the risk of

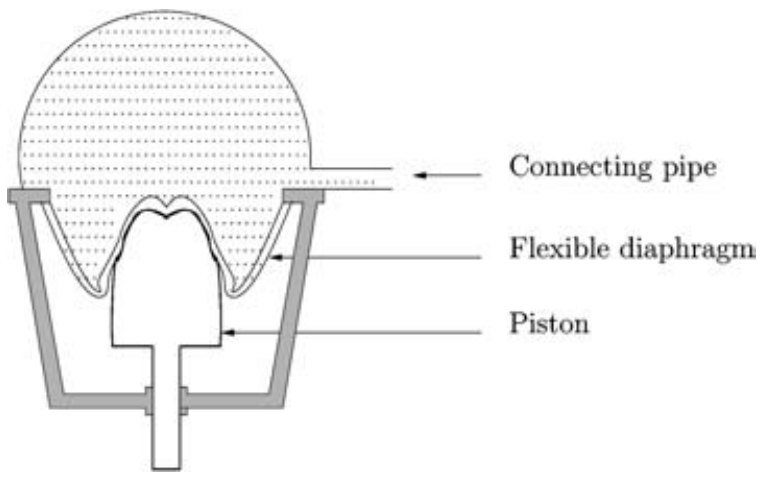

Fig. 4 Sealed cylinder and piston arrangement cavitation (caused by the effective pressure needing to be negative, resulting in 'pulling' on the fluid).

An elementary prototype of the interconnected suspension scheme has been constructed as a benchtop demonstrator (Fig. 5) to illustrate the decoupling property, though it does not attempt to illustrate any dynamic effects. The KCMs are implemented in a conceptually similar way to that of Fig. 3 using pivoted beam arrangements.

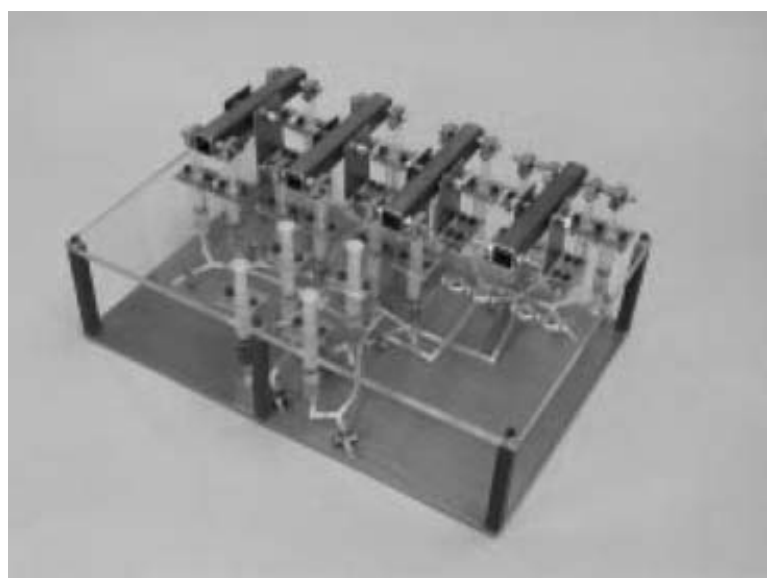

Fig. 5 Prototype interconnected suspension model showing four actuators in the foreground and four KCMs behind 


\subsection{Issues of implementation}

The main goal of this paper has been to introduce a class of interconnected suspensions defined through their four-port admittance matrices [equation (2)] and to show realizability in principle (under ideal modelling assumptions) in two contrasting ways. It needs to be emphasized that each approach to realization will present its own challenges in approximating equation (2) dynamically with sufficient accuracy. For the method described in section 3.5, the use of long interconnection pipes is necessary. It is likely that this will introduce additional damping. Fluid momentum effects may also be of significance at high frequency. In a design for implementation and development it is possible that such effects can be compensated for [e.g. by redesigning the admittances $Q_{i}(s)$ ] or even exploited (e.g. by using the natural damping to eliminate the need for a warp actuator). However, in the present work, it is intended only to point out such issues. They would certainly need to be addressed in any development of a scheme according to the present paper.

\section{GENERALIZATIONS OF THE HYDRAULIC REALIZATION}

\subsection{Two-wheel specialization}

It is straightforward to specialize the class of interconnected suspensions defined in section 2.4 to the two-wheel case. Mathematically, this is defined by an admittance

$$
\mathbf{Y}(s)=\left(\mathbf{T}^{\prime}\right)^{-1} \operatorname{diag}\left(Q_{1}, Q_{2}\right) \mathbf{T}^{-1}
$$

where $\mathbf{T}$ is a real constant non-singular $2 \times 2$ matrix and $Q_{1}(s)$ and $Q_{2}(s)$ are admittances of passive mechanical one-ports. This specialization is suitable for a 'half-car' (two-wheel) vehicle model to study independent adjustment of the bounce and roll/ pitch of the model. In practice, a vehicle might employ such a system for the front suspension while using conventional suspension at the rear, for example.

The realization approach for the four-wheel case shown in Fig. 2 can easily be simplified for the twowheel case, as shown in Fig. 6. The arrangement consists of a four-port mechanical transformer in which ports 1 and 2 are activated by the two wheel-station motions. Two suspension units with admittances $Q_{1}$ and $Q_{2}$ are connected across ports 3 and 4 . A simple choice for $Q_{1}$ and $Q_{2}$ might be a pair of parallel spring-dampers, i.e. $Q_{1}=k_{1} / s+c_{1}$ and $Q_{2}=k_{2} / s+c_{2}$.

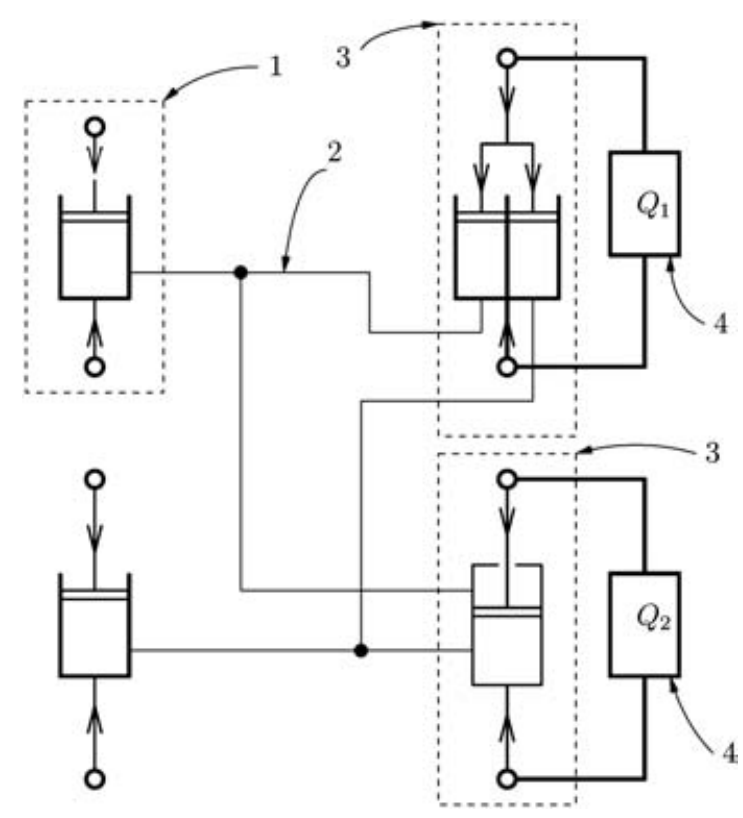

Fig. 6 Interconnected vehicle suspension: two-wheel version (1, actuator; 2 , hydraulic circuit; 3 , kinematic constraint mechanism; 4 , suspension unit)

Such a choice would allow the damping in roll to be specified independently of the damping in bounce. This is not achievable with a suspension using conventional struts and anti-roll bars.

\subsection{Generalization to a six-wheel vehicle}

It is clear that the class of interconnected suspensions defined in section 2.4 may be extended to any number of wheels. As an illustration the case of six wheels is considered with an HGV tractor unit in mind. In the diagram of Fig. 7 the six pistoncylinders on the left-hand side are connected between the respective wheel hubs and the vehicle body in place of the usual suspension arrangement. The six pistons on the right-hand side of the diagram will be called the actuator pistons. The hydraulics part of the arrangement can be thought of as a mechanical transformer between six wheel station pistons and the six actuator pistons. This mechanical transformer itself consists of six separate hydraulic circuits. The implementation is similar in principle to the four-wheel case.

In Fig. 7 a conceptual alternative to the beam arrangement of Fig. 3 for the piston-cylinder kinematic constraints is shown. In this figure, each bank of pistons terminates in a rack, and the two racks mesh into a single cog wheel, thus giving the required kinematic constraint. The impedances $Q_{1}, \ldots, Q_{6}$ are shown connected between the two racks. 


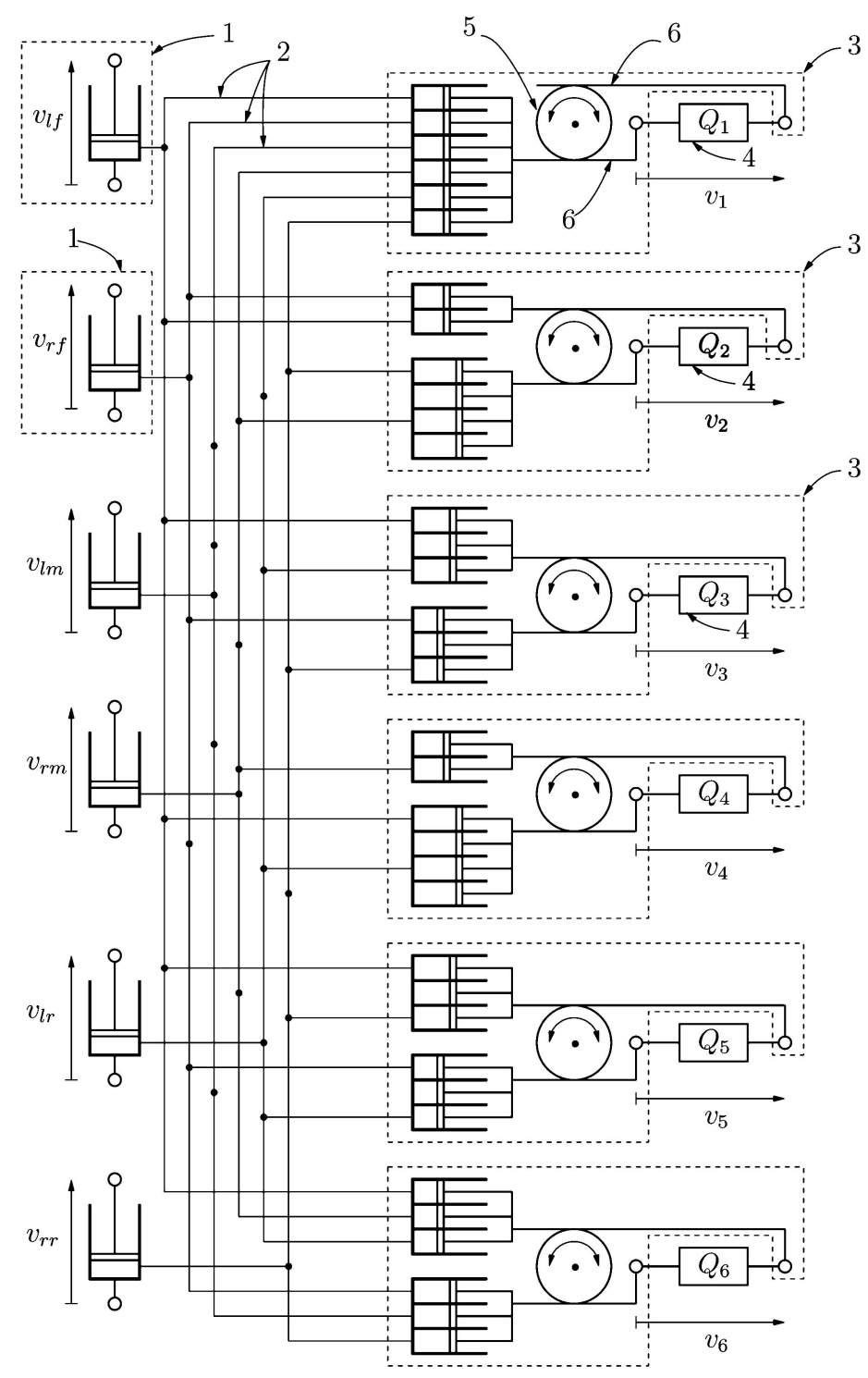

Fig. 7 Interconnected vehicle suspension: six-wheel version (1, actuator; 2 , hydraulic circuit; 3 , kinematic constraint mechanism; 4, suspension unit; 5, cog wheel; 6 , rack)

Following the analysis carried out for the four-wheel case it can be shown that the admittance matrix for this suspension, under appropriate symmetry requirements, takes the form

$$
\mathbf{Y}=\left(\mathbf{T}^{\prime}\right)^{-1} \operatorname{diag}\left(Q_{1}, Q_{2}, Q_{3}, Q_{4}, Q_{5}, Q_{6}\right) \mathbf{T}^{-1}
$$

where

$$
\mathbf{T}=\left[\begin{array}{rrrrrr}
-\lambda_{1} & \lambda_{4} & \lambda_{7} & \lambda_{10} & \lambda_{13} & \lambda_{16} \\
-\lambda_{1} & \lambda_{4} & -\lambda_{7} & \lambda_{10} & -\lambda_{13} & -\lambda_{16} \\
-\lambda_{2} & -\lambda_{5} & \lambda_{8} & -\lambda_{11} & -\lambda_{14} & -\lambda_{17} \\
-\lambda_{2} & -\lambda_{5} & -\lambda_{8} & -\lambda_{11} & \lambda_{14} & \lambda_{17} \\
-\lambda_{3} & -\lambda_{6} & \lambda_{9} & \lambda_{12} & -\lambda_{15} & \lambda_{18} \\
-\lambda_{3} & -\lambda_{6} & -\lambda_{9} & \lambda_{12} & \lambda_{15} & -\lambda_{18}
\end{array}\right]
$$

Observe that the columns of the $\mathbf{T}$ matrix can be associated with modes that might be termed bounce, pitch, roll, undulation, warp, and buckle motions. The ratio $\lambda_{1} / \lambda_{2} / \lambda_{3}$ represents a front-middle-rear weighting for the bounce motion, and similarly with the remaining constants. Note that it is possible to set some of the constants to zero (thus eliminating some of the hydraulic connections) while still retaining much greater flexibility than a conventional suspension; e.g. $\lambda_{10}=\lambda_{13}=\lambda_{16}=0$ could be set.

It should be noted that the suspension arrangement proposed offers the advantage of using 'soft' admittances (e.g. a soft spring-damper parallel combination) at the undulation, warp, and buckle actuator ports. In principle that allows the possibility to significantly soften the overall suspension while retaining control over bounce, pitch, and roll. 


\section{MODULAR REALIZATION OF THE INTERCONNECTED SUSPENSION}

This section describes an alternative approach to realize the class of interconnected suspensions described in section 2.4 using a modular structure for the interconnections. Fig. 8 shows a general schematic of the main suspension unit for such a scheme with four suspension subsystems.

In Fig. 8 the main suspension unit is shown with four mechanical input points whose displacements are $x_{1}, x_{2}, x_{3}$, and $x_{4}$. These points are envisaged to be connected to four actuator locations so that the relative displacement between the vehicle body and each wheel hub is proportional to these displacements. More specifically, the relative displacements at the left-front, right-front, left-rear, and right-rear wheel stations are proportional to $x_{1}, x_{2}, x_{3}$, and $x_{4}$.

To illustrate the idea, consider the case where the suspension subsystem 1 implements the relation

$$
\left(\begin{array}{l}
x_{5} \\
x_{6}
\end{array}\right)=\left(\begin{array}{cc}
\frac{1}{2} & \frac{1}{2} \\
\frac{1}{2} & -\frac{1}{2}
\end{array}\right)\left(\begin{array}{l}
x_{1} \\
x_{2}
\end{array}\right)
$$

or equivalently

$$
\left(\begin{array}{l}
x_{1} \\
x_{2}
\end{array}\right)=\left(\begin{array}{cc}
1 & 1 \\
1 & -1
\end{array}\right)\left(\begin{array}{l}
x_{5} \\
x_{6}
\end{array}\right)
$$

and suspension subsystem 2 implements

$$
\left(\begin{array}{l}
x_{7} \\
x_{8}
\end{array}\right)=\left(\begin{array}{cc}
\frac{1}{2} & \frac{1}{2} \\
\frac{1}{2} & -\frac{1}{2}
\end{array}\right)\left(\begin{array}{l}
x_{3} \\
x_{4}
\end{array}\right)
$$

or

$$
\left(\begin{array}{l}
x_{3} \\
x_{4}
\end{array}\right)=\left(\begin{array}{cc}
1 & 1 \\
1 & -1
\end{array}\right)\left(\begin{array}{l}
x_{7} \\
x_{8}
\end{array}\right)
$$

and suspension subsystems 3 and 4 provide the relationships

$$
\left(\begin{array}{l}
x_{5} \\
x_{7}
\end{array}\right)=\left(\begin{array}{cc}
\lambda_{1} & \lambda_{3} \\
\lambda_{2} & -\lambda_{4}
\end{array}\right)\left(\begin{array}{l}
x_{9} \\
x_{10}
\end{array}\right) \quad\left(\begin{array}{l}
y_{3} \\
y_{4}
\end{array}\right)=\left(\begin{array}{cc}
\frac{1}{2} & \frac{1}{2} \\
\frac{1}{2} & -\frac{1}{2}
\end{array}\right)\left(\begin{array}{l}
y_{1} \\
y_{2}
\end{array}\right)
$$

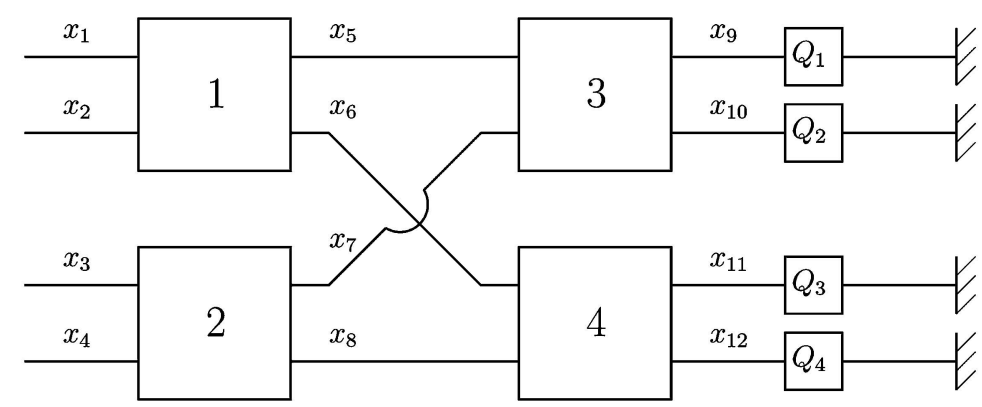

Fig. 8 General schematic

and

$$
\left(\begin{array}{l}
x_{6} \\
x_{8}
\end{array}\right)=\left(\begin{array}{cc}
\lambda_{5} & \lambda_{7} \\
\lambda_{6} & -\lambda_{8}
\end{array}\right)\left(\begin{array}{l}
x_{11} \\
x_{12}
\end{array}\right)
$$
relationship

$$
\left(\begin{array}{l}
x_{1} \\
x_{2} \\
x_{3} \\
x_{4}
\end{array}\right)=\mathbf{T}\left(\begin{array}{l}
x_{9} \\
x_{10} \\
x_{11} \\
x_{12}
\end{array}\right)
$$

where

$$
\mathbf{T}=\left(\begin{array}{cccc}
\lambda_{1} & \lambda_{3} & \lambda_{5} & \lambda_{7} \\
\lambda_{1} & \lambda_{3} & -\lambda_{5} & -\lambda_{7} \\
\lambda_{2} & -\lambda_{4} & \lambda_{6} & -\lambda_{8} \\
\lambda_{2} & -\lambda_{4} & -\lambda_{6} & \lambda_{8}
\end{array}\right)
$$

$$
\left(\mathbf{T}^{\prime}\right)^{-1} \operatorname{diag}\left\{Q_{1}, Q_{2}, Q_{3}, Q_{4}\right\} \mathbf{T}^{-1}
$$
equation (10). points as follows

Combining the above equations gives the overall

It follows that the general form of the mechanical admittance of the suspension system is equal to

where $Q_{1}, Q_{2}, Q_{3}$ and $Q_{4}$ are the mechanical admittances of the suspension units. This is the same admittance that is obtained by the choice of $\mathbf{T}$ in

In Fig. 9, a plan view is given of a mechanism of a suspension subsystem that can implement the above scheme. There are two mechanical input points whose displacements are $y_{1}$ and $y_{2}$, and two mechanical output points whose displacements are $y_{3}$ and $y_{4}$. The mechanism has five sliders and nine links with one fixed pivot. On noting that $y_{5}=-y_{1}$ it can be deduced that the displacements at the output points are related to the displacements at the input 


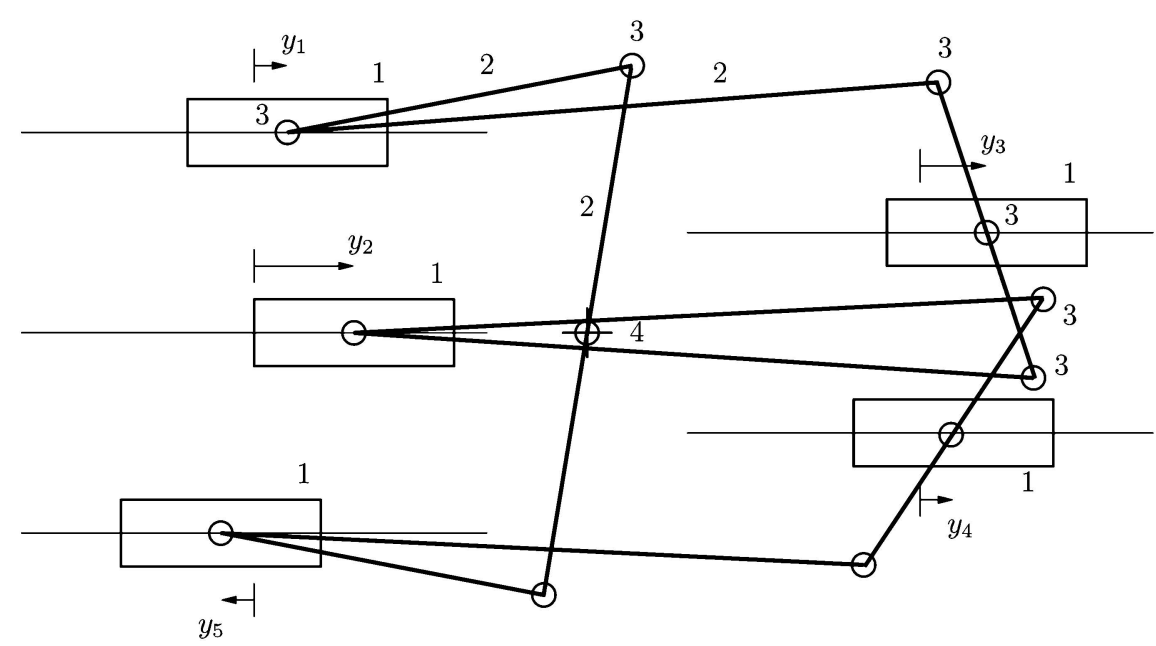

Fig. 9 Plan view of a mechanism of a suspension subsystem (1, slider; 2, link; 3, pivot; 4, fixed pivot)

In Fig. 10, a main suspension unit is shown with four mechanical input points whose displacements are $z_{1}, z_{2}, z_{3}$, and $z_{4}$. The operation is similar to the suspension unit shown in Fig. 8 except for the fact that one of the suspension subsystems is missing. It is envisaged for this arrangement to be applied to a four-wheeled vehicle so that the relative displacements at the left-front, right-front, left-rear, and right-rear wheel stations are proportional to $z_{1}, z_{2}$, $z_{3}$, and $z_{4}$. Thus $z_{5}$ and $z_{7}$ represent the front and rear components of bounce and are connected directly to the suspension elements $Q_{1}$ and $Q_{2}$ without a further transformation into full-car bounce and pitch components. The front and rear components of roll, $z_{6}$ and $z_{8}$, are still transformed into full-car roll and warp components through the suspension subsystem 4 in order to gain the advantage of stiffness in roll and softness in warp. Figure 10 also shows the possibility of an element $\tau$ that directly connects $z_{5}$ and $z_{7}$, e.g. a torsion bar to serve as an anti-pitch bar in order to stiffen pitch but not bounce.

\section{CONCLUSIONS}

This paper has introduced a class of passive interconnected suspensions, defined mathematically in terms of their mechanical admittance matrices, which has increased possibilities to decouple the modes of motion in a vehicle. Some of the advantages of this class are listed as follows:

1. Independently adjustable damping/spring rates for roll, pitch, bounce, and warp are possible, in particular, (arbitrarily) low warp stiffness, independent of bounce, pitch, and roll behaviour.

2. Front-rear weighting may be adjusted independently for bounce, pitch, roll, and warp.

3. Increasing pitch stiffness (independent of bounce and roll behaviour) is just as easy to achieve as increasing roll stiffness, etc.

The independent adjustment of front-rear roll balance alongside low warp stiffness is thought to be a particular advantage for this class, in contrast to

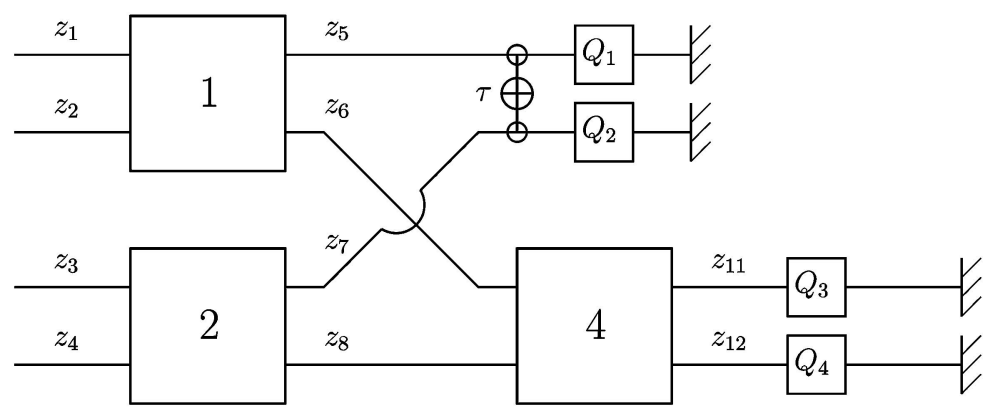

Fig. 10 Simplified schematic 
some simpler approaches where low warp stiffness is achieved at the expense of roll resistance being provided at only one end (front or rear) of the car.

Two alternative realization methods are described for this class. The first employs four interconnected hydraulic circuits to achieve the appropriate decoupling. It was shown that this scheme could be implemented with the suspension units and kinematic constraint mechanisms at a single location on the vehicle, which could be termed the central suspension unit. The second approach allows a modular implementation with mixed mechanical or hydraulic subsystems.

The two realization methods described are intended as a proof of realizability (in principle) for the class of interconnected suspensions introduced in the paper. For any specific manner of implementation, various practical issues would need to be considered, e.g. damping and fluid inertia effects in hydraulic interconnections, packaging, as well as a trade-off between complexity and performance advantage.

The authors believe that advantages are to be gained by introducing a general class of interconnected suspension systems (defined by their dynamic properties as ideal mechanical impedances or admittances), as a step in identifying favourable and unfavourable structures and properties of interconnection. Further work is ongoing to determine quantitatively the performance advantages that the class can provide in the context of a full-car vehicle model and standard performance measures.

\section{ACKNOWLEDGEMENTS}

The authors are grateful to Nicholas Mace and the anonymous reviewers for comments on an earlier draft of this paper. The work was supported in part by the Engineering and Physical Sciences Research Council.

\section{REFERENCES}

1 Ortiz, M. Principles of interconnected suspension. Part 1: modal suspension analysis. Racecar Engineer, 1997, 7(7), 56-59.
2 Mercier, P. E. Vehicle suspensions: a theory and analysis that accord with experiment. Automobile Engineer, 1942, October 405-410.

3 Bastow, D. Car Suspension and Handling, 1993 (Pentech Press, London).

4 Ortiz, M. Principles of interconnected suspension. Part 2: methods of non-rigid interconnection and their effects. Racecar Engineer, 1997, 7(8), 76-81.

5 Pitcher, R. H., Hillel, H., and Curtis, C. H. Hydraulic suspensions with particular reference to public service vehicles. In Public Service Vehicles Conference, IMechE Publications C138, 1977 (Institution of Mechanical Engineers, London).

6 Zapletal, E. Balanced suspension. Racecar Engineer, 2000, 10(2), 41-47.

7 Heyring, C. B. Vehicle suspension system. US Pat. 5,480,188, 2 January 1996.

8 Williams, R. A., Best, A., and Crawford, I. L. Refined low frequency active suspension. In International Conference on Vehicle Ride and Handling, IMechE Publication, 1993, pp. 285-300 (Institution of Mechanical Engineers, London).

9 Smith, M. C. and Walker, G. W. Performance limitations and constraints for active and passive suspension: a mechanical multi-port approach. Veh. System Dynamics, 2000, 33, 137-168.

10 Smith, M. C. and Wang, F.-C. Controller parameterization for disturbance response decoupling: application to vehicle active suspension control. IEEE Trans. Control System Technol., 2002, 10, 393-407.

11 Hayakawa, K., Matsumoto, K., Yamashita, M., Suzuki, Y., Fujimori, K., and Kimura, H. Robust $H_{\infty}$ feedback control of decoupled automobile active suspension systems. IEEE Trans. Automat. Control, 1999, 44(2), 392-396.

12 Shearer, J. L., Murphy, A. T., and Richardson, H. H. Introduction to System Dynamics, 1967 (AddisonWesley, Reading, Massachusetts).

13 Mace, N. and Smith, M. C. Analysis and synthesis of passive interconnected vehicle suspensions, 2004 (in preparation).

\section{APPENDIX}

\section{Notation}

$x, v, F$

lf, rf, lr, rr

displacement, velocity, force

left-front, right-front, left-rear, right-rear (subscripts)

$s \quad$ Laplace transform variable

$\mathbf{Y}(s) \quad$ admittance matrix 